\title{
APLICACIÓN DEL ÍNDICE INTEGRADO RELATIVO DE ANTROPIZACIÓN (INRA), EN UN ECOSISTEMA DE ALTA MONTAÑA
}

\author{
Vianny Plaza-Ortega \\ Mónica Patricia Valencia-Rojas (iD ${ }^{2}$ \\ Apolinar Figueroa-Casas
}

Recibido el 5 de noviembre de 2015, aprobado el 11 de mayo de 2016 y actualizado el 13 de diciembre de 2016

DOI: 10.17151/luaz.2017.44.6

\section{RESUMEN}

Teniendo en cuenta que los ecosistemas de alta montaña no son ajenos al establecimiento de actividades antrópicas, el objetivo de la investigación fue estimar el nivel de antropización en un sistema lagunar de páramo ubicado en el Parque Nacional Natural Puracé, mediante la adaptación del Índice Integrado Relativo de Antropización (INRA). Para ello se seleccionó una ventana de estudio de $6 \times 5 \mathrm{Km}^{2}$ dentro del sistema lagunar a través de herramientas SIG, la cual se dividió en Unidades de Análisis (UA) de $0.5 \mathrm{Km}^{2}$, cada UA contenía a su vez Subunidades de Análisis (SUA) de $0.1 \mathrm{Km}^{2}$ donde se poligonizó las diferentes coberturas de suelo (naturales y antropizadas) encontradas en el ecosistema y a las que se les asignó un valor de 0 a 1, de acuerdo a la escala del índice. Los resultados obtenidos con la aplicación del INRA, destacaron que la categoría de evaluación más frecuente fue la Coberturas sin intervención antrópicadenominada A $(69,51 \%)$, seguido de la categoría Bosques sin intervención antrópica denominada B $(17,29 \%)$. La categoría relacionada con Zonas de pastoreo libre, fue la más baja $(0,97 \%)$; mientras que las categorías Bosques con intervención antrópica (Quema y Pastoreo) e Infraestructura presentaron valores de $4,17 \%$ y $8,02 \%$, respectivamente. Estos resultados permiten concluir que el sistema lagunar presenta un bajo nivel de antropización lo que evidencia que las actividades antrópicas en la zona no generan un impacto marcado para este ecosistema, debido a la poca frecuencia y magnitud de estas actividades, lo que favorece que el ecosistema mantenga estables sus funciones y dinámica.

PALABRAS CLAVE: antropización, índice, páramo.

\section{RELATIVE INTEGRATED ANTHROPIZATION INDEX (INRA) APPLICATION IN A HIGH MOUNTAIN ECOSYSTEM}

\begin{abstract}
Given the high mountain ecosystems are no strangers to the establishment of anthropic activities, the objective of the research was to estimate the level of anthropization in a wasteland lagoon system located in the Puracé National and Natural Park, by adapting the Relative Integrated Anthropization Index (INRA). To this end, a study window of $6 \times 5 \mathrm{Km} 2$ within the lagoon system was selected through GIS tools, which was divided in Units of Analysis (UA, from the Spanish acronym) of $0.5 \mathrm{Km} 2$, each UA contains Subunits of Analysis (SUA, from the Spanish acronym) of $0.1 \mathrm{Km} 2$ where different land cover (natural and anthropic) found in the ecosystem were polygonized and assigned a value of 0 to 1 , according to the index scale. The results obtained with the application of RIAI, emphasized that the most frequent assessment category was the Cover without human intervention called A (69.51\%), followed by the category Forests without human
\end{abstract}


intervention called B (17.29\%). The category related to free grazing Zones was the lowest $(0.97 \%)$ while the Forests categories with anthropic intervention (Burning and grazing) and Infrastructure presented values of $4.17 \%$ and $8.02 \%$, respectively. These results suggest that the lagoon system has a low level of anthropization which evidences that anthropic activities in the area do not generate a strong impact on the ecosystem due to their low frequency and magnitude, thus making possible to keep stable the ecosystem functions and dynamics.

KEYWORDS: anthropization, index, páramo.

\section{INTRODUCCIÓN}

La región andina colombiana es una de las regiones más afectadas por procesos de transformación antrópica (Armenteras, Gast \& Villareal, 2003; Bernsen, 1991) dado su aspecto biofísico que la han convertido en terrenos propicios para los asentamientos humanos y para el desarrollo de actividades que favorecen la economía de sus habitantes. Sobre la región andina colombiana se encuentran ecosistemas estratégicos como los páramos o ecosistemas de alta montaña, los cuales están protegidos por la legislación nacional (Ley 99/1993; resolución 769/2002, Plan Nacional de Desarrollo 2010-1014, Política Nacional de Humedales Interiores, entre otras); sin embargo, debido a los patrones de distribución de la población colombiana sobre esta región del país, estos ecosistemas son amenazados y vulnerados por procesos de transformación en el paisaje, paramización y actividades humanas (Armenteras et al., 2003; MA, 2002), cuyos efectos negativos se ven representados principalmente en deterioro ambiental (pérdida de hábitats nativos y diversidad, erosión y cambios en el uso del suelo) (Hofstede, 1995; Hofstede, Segarra \& Mena V, 2003; MAVDT \& IAvH, 2010; Molinillo \& Monasterio, 2002; J. Morales \& Estévez, 2006; van der Hammen, 2008; Vargas, Premauer \& Cárdenas, 2002) e impactos en el ciclo del agua, ya que alteran significativamente el comportamiento hidrológico, las propiedades físicas y químicas, la función de suministro y el desarrollo de la biota acuática (Buytaert et al., 2006; Ruíz, 2009).

Debido al paso acelerado de las actividades humanas sobre ecosistemas de alta montaña, instituciones e investigadores han aunado esfuerzos para caracterizar y monitorear estos ambientes con el fin de conocer el estado de conservación de sistemas lagunares, lagos y humedales a partir de diferentes factores de evaluación. Sin embargo y pese al avance en los estudios limnológicos (Castro, 2009; Donato, 2001; Imbachí, 2014; S. Morales \& Salazar, 2012; Múñoz, 2008; G. Pinilla, 2010; G. A. Pinilla et al., 2010; Plaza, 2014; Roldán-Pérez, 2009; Roldán, 2003; Roldán \& Ramírez, 2008; Toro, Jaramillo, Ocampo, Correa \& Salgado, 2012), aún para el país son escasos los estudios integrales que permiten evaluar cómo las actividades antrópicas ejercen presión sobre las funciones y dinámicas ecosistémicas de estos ambientes acuáticos.

Esta investigación se desarrolló en el sector San Rafael (Parque Nacional Natural Puracé), el cual es un importante elemento cultural para el resguardo indígena de Puracé por considerarse sitio sagrado para su comunidad, donde se llevan a cabo rituales de refrescamiento y limpias ${ }^{a}$; ecológicamente este sistema es importante para el Parque Nacional Natural Puracé, pues provee de diferentes servicios ecosistémicos a la zona y además sirve de hábitat de especies endémicas y amenazadas de la región. Pese a su importancia, este sector ha sufrido procesos de degradación antrópica tales como pastoreo y pesca principalmente (Joaqui, 2005; J. Martínez, 2005; Mosquera, 2009; Mosquera, Martínez \& Figueroa, 2014; Múñoz, 2008; Múñoz, 
Figueroa \& Vergara, 2009; Plaza, 2014). A partir de lo anterior, se realizó una evaluación en la cual se adaptó la aplicación del índice integrado relativo de antropización (INRA) a partir de la propuesta de Martínez (2010) con el fin de conocer la antropización de manera cuantitativa y cualitativa, a través del cambio en los tipos de suelo de las unidades de análisis que componen una ventana de estudio.

\section{MATERIALES Y MÉTODOS}

\section{Área de estudio}

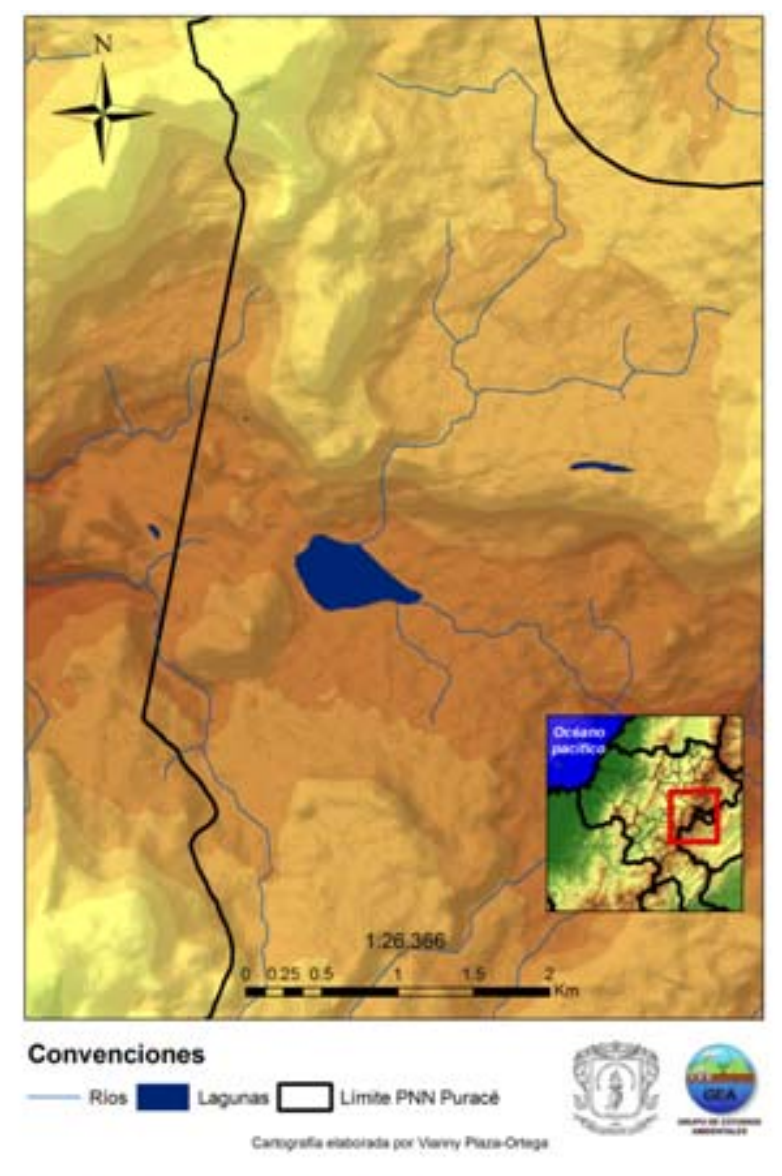

Fuente: Grupo de Estudios Ambientales

Figura 1. Área de Estudio.

El estudio se realizó en el sistema lagunar de San Rafael el cual se encuentra ubicado en una zona de transición entre el Parque Nacional Natural Puracé y el resguardo indígena de Puracé a 3300 m.s.n.m. en un valle aluvial definido como una zona de vida paramuna (UAESPNN, 2004) (Figura 1); debido al traslape de territorios, el sistema lagunar conjuga actividades de manejo, conservación y subsistencia como la pesca tradicional y el pastoreo, siendo esta última la que más ejerce presión en el sistema debido a la descomposición de la materia orgánica y el efecto del pisoteo constante. Hasta ahora, la consecuencia más notable en el sistema lagunar ha sido la reducción del espejo de agua, el cual ha disminuido a través de los años. Yasnó et al. (2000) reportó un área de 26.5 ha (citado por Joaquí, 2005); Donato (2001) reportó 20 ha; mientras que Muñoz (2008) reportó un área aproximada de 18.9 ha para el espejo de 
agua, a partir de la poligonización del espejo de agua de la laguna. Actualmente, la laguna tiene un área de 16,75 ha aproximadamente (Plaza, 2014).

\section{Métodología}

A partir de la propuesta metodológica de Martínez (2010), se adaptó la aplicación del INRA para esta investigación, de acuerdo con las coberturas vegetales propias del páramo (arbóreo, arbustales, humedal, laguna y pajonal-frailejonal), identificadas previamente en los trabajos de Mosquera (2009), Muñoz (2008) y Joaquí (2005) de la siguiente manera:

- Cambio en la definición de las coberturas o usos del suelo: esta modificación se realizó teniendo en cuenta que el ecosistema abordado en la investigación es un ecosistema de páramo, mientras que en el trabajo de Martínez la aplicación del INRA se realizó en un ecosistema urbano en la meseta de Popayán a 1750-1900 m.s.n.m., teniendo en cuenta los rangos altitudinales y las diferencias en las zonas de vida trabajada, se encuentran coberturas vegetales diferentes en composición y estructura a las trabajadas por Martínez.

- Reducción de las subunidades de análisis (SUA): la cual se considera a partir del nivel de detalle al que se pretende acceder en la investigación; cabe resaltar que este apartado es comentado y sugerido inicialmente por Martínez (2010).

- Cambio en las categorías de evaluación: las cuales se definen a partir de las coberturas o usos de suelo encontrados en el ecosistema trabajado.

\section{Aplicación del INRA}

Para el desarrollo de esta investigación se recogió información secundaria basada en revisiones literarias de estudios realizados en ecosistemas de páramo a escala nacional y local, con el fin de identificar las coberturas vegetales más representativas de estos ecosistemas y un registro de las actividades antrópicas más recurrentes en la alta montaña colombiana (Figura 2).

Una vez identificadas las coberturas y usos de suelo propios de páramo, se les asignó un valor entre 0 y 1 cualitativamente, donde 0 es una cobertura sin ningún tipo de modificación antropogénica y 1 son coberturas intervenidas. Estos valores se asignaron de acuerdo con la intensidad del impacto generado en los ecosistemas de páramo, tomando como referentes los diferentes reportes literarios (Figura 2,Tabla 1).

Por otro lado, a través de las observaciones de campo y talleres con los diferentes actores sociales se identificaron las actividades antrópicas que tienen ocurrencia en el sistema lagunar de San Rafael (pastoreo, quemas ocasionales, pesca tradicional, turismo), los cuales sirvieron de referente para construir la matriz de FEARO e identificar los componentes más afectados por dichas actividades (Plaza, 2014) (Figura 2).

Una vez obtenidas las herramientas de trabajo necesarias (actividades antrópicas, coberturas vegetales o usos de suelo), se realizó el procesamiento de imágenes a través de sistemas de información geográfica (SIG). Para ello se tomó una imagen satelital del año 2010 del área de estudio, con una extensión de $6 \times 5 \mathrm{Km}$, a la que se le hizo el procedimiento de clasificación supervisada mediante el programa ArcMap versión 9.3 (ESRI, 1999-2008). Posteriormente, se poligonizó mediante el programa Global Mapper versión 14.0 (BMG, 2002-2012) las coberturas o usos del suelo de interés relacionadas en la Tabla 1 , y se definieron unidades de análisis (UA) de 
$0,5 \mathrm{Km}^{2}$ por medio de grillas; dentro de cada UA se asignaron subunidades de análisis (SUA) de $0,1 \mathrm{Km}^{2}$, cada una (Figura 2).

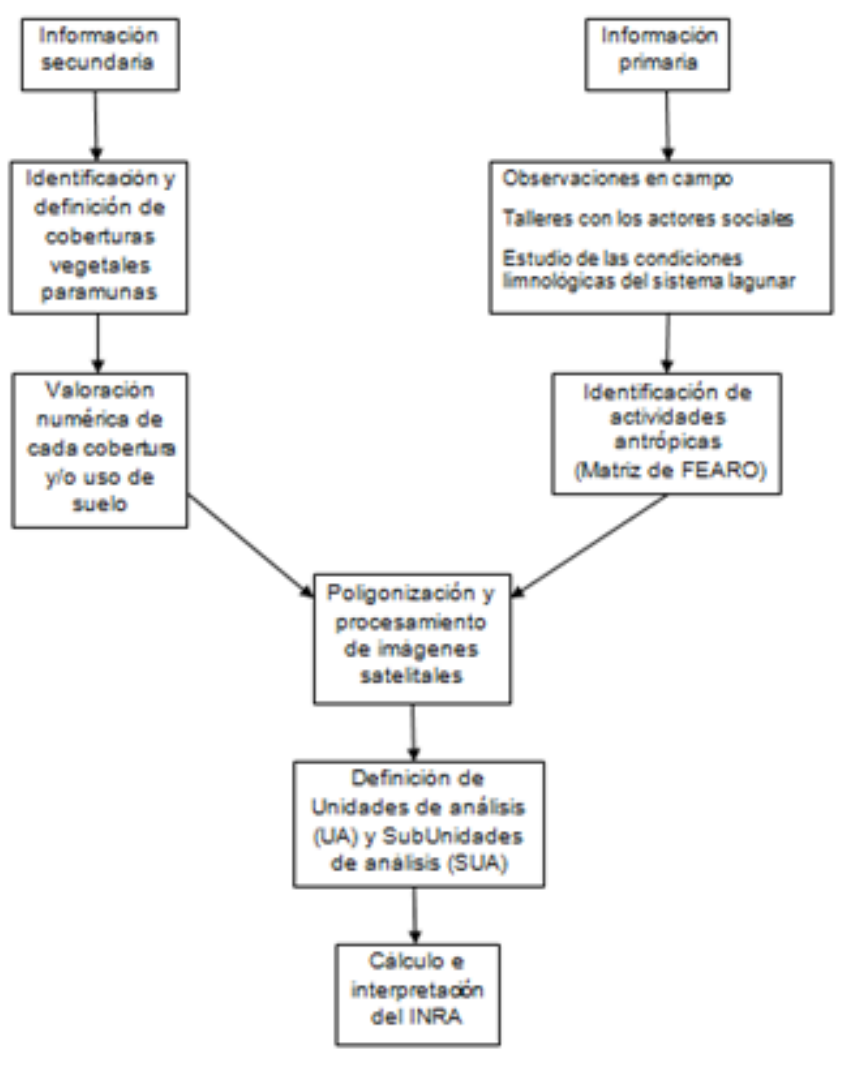

Fuente: Los autores

Figura 2. Esquema metodológico de la adaptación y aplicación del INRA.

El trabajo con las herramientas de información geográfica fue corroborado en campo mediante caminatas y avistamientos en la ventana de estudio. 
Tabla 1. Coberturas/uso de suelo.

\begin{tabular}{|c|c|c|c|c|}
\hline Simbología & $\begin{array}{l}\text { Categoría de } \\
\text { evaluación }\end{array}$ & Definición & $\begin{array}{l}\text { Area } \\
(\mathrm{Ha})\end{array}$ & Valor \\
\hline A & $\begin{array}{l}\text { Coberturas sin } \\
\text { intervención } \\
\text { antrópica }\end{array}$ & $\begin{array}{l}\text { Lagunas, Humedales, } \\
\text { Frailejones, Gramíneas }\end{array}$ & 547.42 & 0 \\
\hline$B$ & $\begin{array}{l}\text { Bosques sin } \\
\text { intervención } \\
\text { antrópica }\end{array}$ & $\begin{array}{l}\text { Coberturas boscosas que } \\
\text { no presentan ningún tipo de } \\
\text { actividad humana }\end{array}$ & 190.070 & 0,25 \\
\hline C & $\begin{array}{l}\text { Bosques con } \\
\text { intervención } \\
\text { antrópica (quemas y } \\
\text { pastoreo) }\end{array}$ & $\begin{array}{l}\text { Coberturas boscosas que } \\
\text { presentan actividades de } \\
\text { quema y pastoreo } \\
\text { recurrentemente }\end{array}$ & 31.95 & 0,5 \\
\hline $\mathrm{D}$ & Zonas de pastoreo & $\begin{array}{l}\text { Zonas libres de pastoreo en } \\
\text { áreas de frailejones y } \\
\text { chuscales }\end{array}$ & 2.22 & 0,75 \\
\hline$E$ & Infraestructura & $\begin{array}{l}\text { Vía principal, camino de } \\
\text { herraduras y casas }\end{array}$ & $\begin{array}{c}0,53 \\
\text { (casas) }\end{array}$ & 1 \\
\hline
\end{tabular}

Finalmente, se calculó el valor del INRA para cada UA, siguiendo la metodología descrita por Martínez (2010), usando la siguiente fórmula:

$$
\mathrm{INRA}^{\prime}=\left(\Sigma \mathrm{SUA}^{\prime} / \mathrm{n}\right) \cdot 100
$$

Donde: $\Sigma$ SUA, es la sumatoria del valor de antropización para todas las SUA y $\mathrm{n}$, es el número total de SUA.

La interpretación final del INRA varía de 0-100, donde 0 son valores de UA con nula antropización y 100 son valores de UA muy antropizados (Figura 2).

\section{RESULTADOS Y DISCUSIÓN}

\section{Caracterización ambiental}

Para el sistema lagunar de San Rafael, se trabajaron cinco tipos de coberturas vegetales (arbóreo, arbustales, humedal, laguna y pajonal-frailejonal) definidas de acuerdo con el trabajo de Mosquera (2009). Entre estas se identificaron especies arbóreas y arbustales de los géneros Polylepis, Pentacalia, Hypericum, Diplostephium, especies Senecio andicola, Hesperomeles lanuginosa, con registros de actividades humanas como pastoreo, quemas, contaminación por residuos sólidos e igualmente un camino de herradura que sirve para el tránsito del ganado.

La cobertura vegetal pajonal-frailejonal se caracterizó por formaciones abiertas de frailejones (Espeletia sp.), gramíneas (Calamagrostis effusa y Cortaderia sp.) y la 
zona de humedal caracterizada por cojines de plantas vasculares (Paepalanthussp.) y musgos (Sphagnum sp. y Breutelia sp.); donde se registró un camino de herradura que sirve de paso para turistas que visitan el cuerpo de agua.

El área del humedal y la laguna presentaron vegetación que se caracterizó por especies herbáceas de la zona litoral (Myriophyllum quitense, Lachemilla paludicola, Potamogeton paramoanus, Isoetes killipii, Ranunculus nubigenus, entre otras) (Plaza, 2014), registrándose actividades de pesca tradicional, refrescamiento y turismo.

La matriz de FEARO arrojó que dentro de las actividades más impactantes para la zona de estudio están el pastoreo, las quemas ocasionales y el turismo (Plaza, 2014). Mediante las observaciones realizadas en campo, resultó evidente que la figura de áreas protegidas, bajo la cual se encuentra el sistema léntico, no ha significado un obstáculo para el desarrollo de dichas actividades, debido a la poca articulación entre los diferentes actores sociales que no ha permitido generar soluciones alternativas para controlar el manejo de las actividades de subsistencia que se dan en la zona de estudio.

\section{Aplicación del índice integrado relativo de antropización}

Las modificaciones que se realizaron para la aplicación del INRA en un ecosistema de alta montaña, permitieron hacer una evaluación de la zona de estudio donde se tuvieron en cuenta las coberturas vegetales propias del páramo. De esta forma, el análisis espacial permite entender cómo los cambios, propiciados por el cambio de uso o coberturas de suelo mediadas por las actividades antrópicas, afectan directamente la estructura del ecosistema, la funcionalidad y servicios ecosistémicos propios que oferta esta zona de vida.

Con la aplicación del INRA se observó que la categoría de evaluación más frecuente fue la denominada A $(69,51 \%)$, seguida de la categoría B $(17,29 \%)$, los cuales corresponden a coberturas y bosques sin ningún tipo de intervención antrópica, respectivamente. La categoría relacionada con zonas de pastoreo libre, fue la más baja $(0,97 \%)$; mientras que las categorías bosques con intervención antrópica (quema y pastoreo) e infraestructura, presentaron valores de $4,17 \%$ y $8,02 \%$, respectivamente. Los valores obtenidos en la aplicación del INRA muestran claramente que en el sistema lagunar de San Rafael predominaron las coberturas sin intervención como: los valles de frailejones, gramíneas, humedales, los cuerpos de agua y los bosques $\sin$ actividad de quema y pastoreo, debido a la geomorfología del terreno y el valor cultural y de conservación que tiene el sistema lagunar para los diferentes actores sociales de la zona.

Sin embargo, en la ventana de estudio, las UA: E6, F5, H6 y H7 (Figura 3) presentaron los valores más altos del INRA, debido a que están relacionados con coberturas de bosques donde se registraron actividades antrópicas, zonas de pastoreo, casas, vías principales y caminos de herradura, lo que otorga el carácter de antropización a estas UA. Las vías de acceso como la carretera hacia La Plata y los caminos de herradura constituyen un aporte a las actividades antrópicas asociadas.

Las vías de conectividad existentes han propiciado el desarrollo de las diferentes actividades humanas que se llevan a cabo en la zona, pues ellas favorecen el tránsito y desplazamiento de animales, turistas y pescadores que buscan obtener un mayor provecho de los servicios que ofrece este ecosistema. Si se compara este análisis con lo obtenido por Joaquí (2005), quien reportó una baja incidencia de áreas intervenidas para este sector, se observa que en la actualidad y como consecuencia de las vías de 
acceso, se han incrementado las áreas intervenidas en la zona de estudio, representadas como zonas de pastoreo y bosques intervenidos (Figura 3).

En general, los valores del INRA para los cuadrantes evaluados, oscilaron entre 0 y 12,25 , lo que confirma que el paisaje evaluado presenta valores muy bajos de antropización en la escala del índice (Figura 3); sin embargo, cuando se tiene en cuenta la fragilidad del ecosistema y su lenta capacidad de resiliencia, los valores obtenidos toman una interpretación diferente, pues los efectos de las actividades antrópicas en ecosistemas de páramos son más drásticos si se compara con otros ecosistemas de zonas más bajas en la región andina (p.ej.: bosques andinos), debido a que los procesos de recuperación de estos ecosistemas son más lentos por influencia de sus condiciones biofísicas (Cleef, 2008; J. Morales \& Estévez, 2006). Por tal razón, los impactos generados por estas actividades comprometen fuertemente la dinámica y servicios ecosistémicos que prestan estos ecosistemas estratégicos, ya que propician procesos de transformación, degradación y pérdida de las propiedades de los elementos del paisaje en una escala temporal (J. Morales \& Estévez, 2006; Serrano Evers, Páez Ramírez \& Kolter Arrieta, 2008).

Los valores bajos obtenidos en la escala de interpretación del índice pueden ser explicados debido a que las actividades antrópicas identificadas durante el muestreo no son recurrentes ni intensivas en la zona; sin embargo, cuando se contrasta con la literatura, las investigaciones previas multitemporales (J. Martínez, 2005; Mosquera, 2009; Vidal, 2012) evidencian procesos de transformación y degradación que han sido mediados por distintas actividades antrópicas que han ocurrido a lo largo del tiempo y han sido generadas por procesos de ocupación del territorio, cuyos impactos y afectaciones ocurren sobre una línea de tiempo más amplia, sugiriendo de esta manera estudios multitemporales en la aplicación del INRA. 

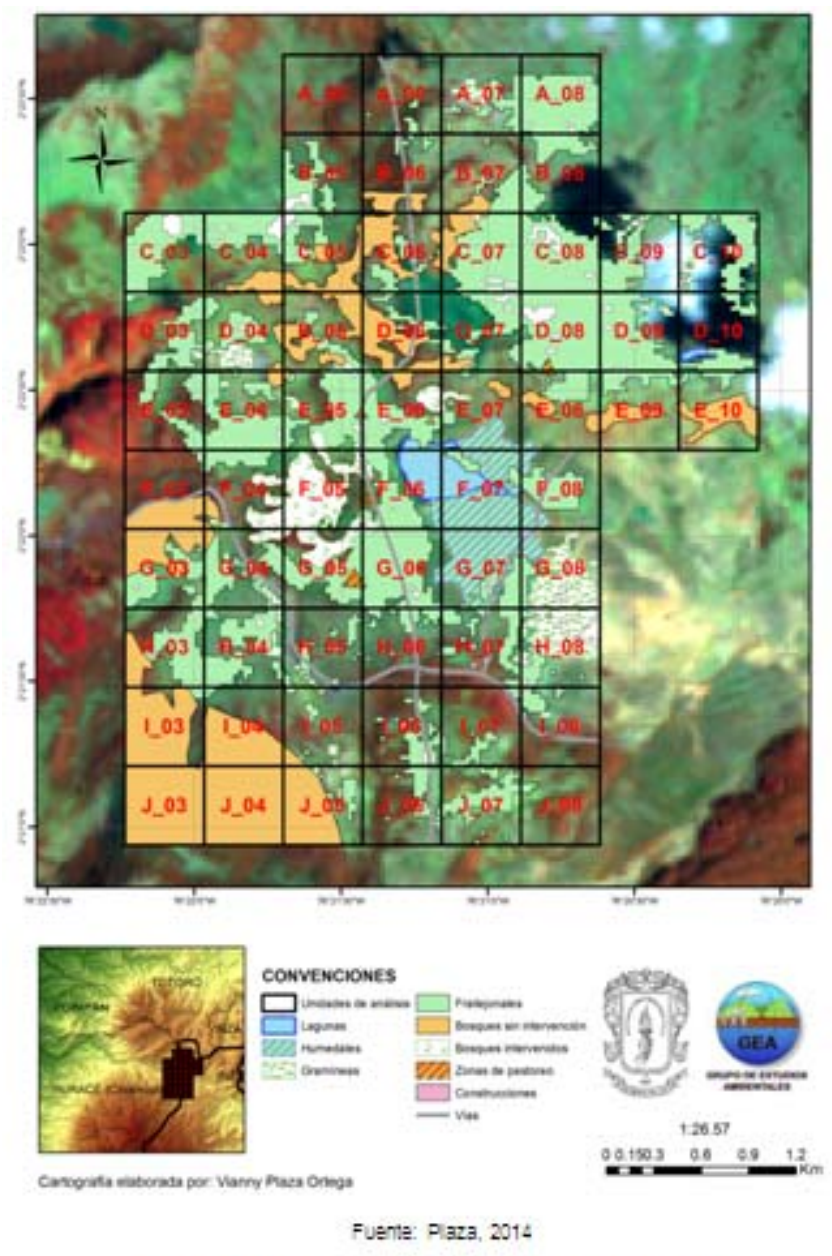

Figurs 3. Maps de coberturss de suelo pars INRA.

\section{CONCLUSIONES}

Las modificaciones que se realizaron al INRA con el fin de ser aplicado sobre un ecosistema de alta montaña permitieron estimar la antropización del sistema lagunar desde sus condiciones actuales de uso y cobertura del suelo, lo que demuestra la flexibilidad de aplicación del INRA, dado su carácter relativo e integral. La interpretación que de éste se desprende sugiere involucrar la historia natural del ecosistema con el fin de contextualizar los resultados que se obtengan a partir de dicha aplicación.

Aunque en general, la aplicación del índice arrojó que el sistema lagunar de San Rafael presenta un bajo nivel de intervención antrópica, las actividades antrópicas que se han desarrollado producto del proceso de ocupación del territorio, tienen impactos importantes sobre este ecosistema estratégico debido a su lenta capacidad de recuperación. Si bien las actividades antrópicas que se desarrollan en la actualidad distan de ser recurrentes e intensivas y no generan cambios inmediatos tan marcados, esta investigación constituye un elemento de soporte para proponer medidas de planificación que promuevan la conservación y el uso sostenible del ecosistema. 
EI INRA constituye una herramienta que aporta al entendimiento de la antropización a través de un enfoque paisajístico; por eso es importante aplicar el índice en estudios multitemporales con el fin de dimensionar y estimar la antropización que se ha generado en un ecosistema como consecuencia de la ocupación y los procesos de transformación que han tenido lugar en él a través del tiempo.

\section{AGRADECIMIENTOS}

Agradecemos al Resguardo Indígena de Puracé, al Parque Nacional Natural Puracé y sus funcionarios, a los auxiliares de campo, a Héctor Ramírez y Samir Joaquí por los aportes y revisiones del documento, a Carlos Gúzman y Dayán González del Grupo de Estudios Ambientales por el apoyo con las herramientas SIG. De igual forma, agradecemos a la Vicerrectoría de Investigaciones de la Universidad del Cauca, el Grupo de Estudios Ambientales y el Doctorado en Ciencias Ambientales de la alma mater por el apoyo financiero para la realización del trabajo de grado del cual se derivó esta investigación.

\section{POTENCIAL CONFLICTO DE INTERESES}

La autora principal manifiesta que no existe ningún tipo de conflicto de intereses respecto a la publicación de este artículo, el cual se deriva del trabajo de pregrado de la misma autora, bajo el asesoramiento de la Ph. D Mónica Valencia Rojas y la dirección del Ph. D Apolinar Figueroa Casas.

\section{REFERENCIAS}

- Armenteras, D., Gast, F. \& Villareal, H. (2003). Andean forest fragmentation and the representativeness of protected natural areas in the eastern Andes, Colombia. Biological Conservation, 113(2), 245-256.

- Bernsen, O. (1991). Observaciones preliminares sobre cultivo en zonas de páramo de Colombia. Novedades colombianas, Nueva Epoca, 3, 63-73.

- $\quad$ BMG. (2002-2012). Global Mapper (Version 14.0).

- Buytaert, W., Célleri, R., De Bièvre, B., Cisneros, F., Wyseure, G., Deckers, J. \& Hofstede, R. (2006). Human impact on the hydrology of the Andean páramos.EarthScience Reviews, 79(1-2), 53-72.

- Castro, D. (2009). Desarrollo de un índice de diatomeas perifíticas para evaluar el estado de los humedales bogotanos. ( Tesis de Maestría ), Universidad Nacional de Colombia, Bogotá. 
- Cleef, A. M. (2008). Influencia humana en los páramos. In C. C. Serrano Evers, A. Páez \& L. Kolter Arrieta (Eds.), Memorias Panorama y perspectivas sobre la gestión ambiental de los ecosistemas de páramo. (pp. 26-33). Bogotá: Procuraduria General de la Nación.

- Donato, J. C. (2001). Fitoplancton de los lagos andinos del norte de sudámerica (Colombia). Santafe de Bogotá, D.C. Colombia: Academia Colombiana de Ciencias Exactas, Físicas y Naturales.

- Donato Rondón, J. C., González G, L. E. \& Rodríguez M, C. L. (1996). Ecología de dos sistemas acuáticos de páramo (Vol. 9). Santafe de Bogotá, D.C. Colombia: Academia Colombiana de Ciencias Exactas, Físicas y Naturales

- $\quad$ ESRI. (1999-2008). ArcMap (Version 9.3).

- Hofstede, R. (1995). The effects of grazin and burning on soil and plant nutrient concentrations in Colombian páramo grasslands. Plant and Soil, 173, 111-132.

- Hofstede, R., Segarra, P. \& Mena V, P. (2003). Los páramos del mundo. Quito: Global Peatland Initiative/NC-IUCN/Ecociencia.

- Imbachí, D. (2014). Integridad ecológica de la laguna de San Rafael Parque Nacional Natural Puracé, Cauca. (Trabajo de grado), Universidad del Cauca, Popayán.

- Joaqui, S. C. (2005). Análisis multitemporal de las coberturas vegetales para ecotopos paramunos caracterizando las intervenciones antrópicas, en una ventana del Parque Nacional Natural Puracé. (Trabajo de grado), Universidad del Cauca, Popayán.

- MA. (2002). Política nacional de humedales interiores de Colombia. Estrategias para su conservación y uso sostenible. Bogotá D.C

- Martínez, J. (2005). Estudio espacio temporal del proceso de fragmentación en la zona nor-oriental del Parque Nacional Natural Puracé, mediante el análisis de las comunidades vegetales. (Tesis pregrado), Cauca, Popayán.

- Martínez, W. A. (2010). INRA- Índice Integrado Relativo de Antropización: Propuesta técnica-conceptual y aplicación. Revista Intropica, 5(1), 45-54.

- MAVDT \& IAvH. (2010). Definición de criterios para la delimitación de páramos del país y de lineamientos para su conservación. PARTE I (pp. 91). Bogotá: 
- Ministerio de Ambiente, Vivienda y Desarrollo Territorial - Instituto de Investigación de Recursos Biologicos Alexander von Humboldt.

- MAVDT \& UN. (2008). Metodología para la estimación del caudal ambiental en proyectos licenciados (pp. 135). Bogotá, D.C: Ministerio de Ambiente, Vivienda y Desarrollo Territorial - Universidad Nacional de Colombia.

- Molinillo, M. \& Monasterio, m. (2002). Patrones de vegetación y pastoreo en ambientes de páramo. Ecotrópicos, 15(1), 19-34.

- Morales, J. \& Estévez, J. (2006). El páramo: ¿ecosistema en vía de extinción?Revista Luna azul, 1(22), 39-51.

- Morales, S. \& Salazar, M. (2012). Diatomeas perifíticas de lagos con diferente estado trófico en el departamento del Cauca. Luna Azul, 35, 10-27.

- Mosquera, A. (2009). Caracterización de dos zonas de transición, mediante el análisis de las coberturas vegetales y variables microambientales en una zona de páramo, en el sector nororiental del Parque Nacional Natural Puracé. (Tesis de pregrado), Universidad del Cauca, Popayán.

- Mosquera, A., Martínez, J. \& Figueroa, A. (2014). Microclimatic gradients in transition zones of Andean forest: A case study of Purace National Park.Scientific Research and Essays, 9(16), 703-715.

- Múñoz, F. (2008). Caracterización biofísica, análisis espacio temporal y de intervenciones antrópicas, para humedales altoandinos, caso tipo laguna de San Rafael (Zona Norte) Parque Nacional Natural Puracé y Humedal de Calvache. (Tesis de Pregrado), Universidad del Cauca, Popayán-Cauca.

- Múñoz, F., Figueroa, A. \& Vergara, H. (2009). Análisis espacio temporal de humedales altoandinos: laguna de San Rafael y humedal de Calvache. In A. Figueroa \& M. Valencia (Eds.), Fragmentación y coberturas vegetales en ecosistemas andinos, departamento del Cauca (pp. 157-188). Popayán: Universidad del Cauca.

- Pinilla, G. (2010). An index of limnological conditions for urban wetlands of Bogota city, Colombia. Ecological Indicators, 10(4), 848-856.

- Pinilla, G. A., Duarte, J. \& Vega, L. (2010). Índice de Estado Limnológico (IEL) para evaluar las condiciones ecológicas de las ciénagas del canal del Dique, Colombia. Acta Biológica Colombiana, 15(2), 169-188. 
- Plaza, V. (2014). Alteraciones antrópicas en el sistema lagunar San Rafael (Parque Nacional Natural Puracé) a través de tres subdivisiones de hábitat. (Trabajo de grado), Universidad del Cauca, Popayán.

- Roldán-Pérez, G. (2009). Desarrollo de la limnología en Colombia: Cuatro décadas de avances progresivos. Actual Biol, 31(91), 227-237.

- Roldán, G. A. (2003). Bioindicación de la calidad del agua en Colombia. Uso del método BMWP/Col. Medellín: Universidad de Antioquia.

- Roldán, G. A. \& Ramírez, J. J. (2008). Fundamentos de limnología neotropical (2 ed.): Academía de Ciencias Exactas Físicas y Naturales.

- Ruíz, D. M. (2009). Determinación del impacto ambiental sobre el recurso hídrico para consumo humano generado por el establecimiento de actividades antrópicas en la parte alta de la subcuenca río San Francisco, Municipio Puracé. Departamento del Cauca. (Tesis de Pregrado), Universidad del Cauca Popayán.

- Serrano Evers, C. C., Páez Ramírez, A. \& Kolter Arrieta, L. (2008). Situación de los páramos en Colombia frente a la actividad antrópica y el cambio climático (P. D. p. A. A. y. Agrarios, Trans.). In 4 (Ed.), Coleccion asuntos ambientales (pp. 112). Bogotá: Instituto de Estudios del Ministerio Público.

- Toro, D. R., Jaramillo, M. T., Ocampo, D. M., Correa, R. M. \& Salgado, P. A. (2012). Estudio limnológico de la Laguna Negra. Zona amortiguadora del P.N.N Los Nevados. Boletin Cientifico Centro de museos museo de historia nacional, 16(2), 23-38.

- UAESPNN. (2004). Plan de manejo Parque Nacional Natural Puracé. Popayán.

- van der Hammen, T. (2008). El páramo: de la destrucción a la conservaciónMemorias Panorama y perspectivas sobre la gestión ambiental de los ecosistemas de páramo. (pp. 11-15): Procuraduria General de la Nación.

- Vargas, O., Premauer, J. \& Cárdenas, C. d. I. Á. (2002). Efecto del pastoreo sobre la estructura de la vegetación en un páramo húmedo de Colombia.Ecotrópicos Sociedad Venezolana de Ecología, 15(1), 35-50.

- Vidal, C. C. (2012). Relación de procesos de fragmentación ecosistémica con diversidad de comunidades de aves frugivoras e insectivoras en dos sectores altoandinos de la cordillera central en el departamento del Cauca(Tesis de pregrado), Universidad del Cauca, Popayán. 
1. Bióloga. Grupo de Estudios Ambientales, Museo de Historia Natural de la Universidad del Cauca. Tel (+57 2) 8209800 ext: 2607. viviluna18@gmail.com(Autor de correspondencia). Identificación ORCID 0000-0003-0886-1393

2. Ph.D en Ciencias ambientales. Grupo de Estudios Ambientales, Museo de Historia Natural de la Universidad del Cauca. Tel (+57 2) 8209800 ext: 2607.mpvalenciar@gmail.com Identificación ORCID 0000-0002-7483-2235

3. Ph.D en Ciencias biológicas. Profesor titular del Departamento de Biología, Universidad del Cauca.Director Grupo de Estudios Ambientales. Museo de Historia Natural de la Universidad del Cauca. Tel $(+57$ 2) 8209800 ext: 2607.apolinarfigueroa@gmail.com 0000-0003-3586-8187

a. Ritual que se lleva a cabo en la laguna de San Rafael por las autoridades indígenas del resguardo de Puracé, con el fin de realizar una limpia de varas como agradecimiento a las ninfas de las aguas. Igualmente, el ritual también sirve para armonizar la comunidad y obrar con rectitud y equilibrio. La actividad tiene un tiempo de duración hasta pasada la medianoche.

Para citar este artículo: Plaza-Ortega, V.; Valencia-Rojas, M.P. y Figueroa-Casas, A. (2017). Aplicación del índice integrado relativo de antropización (INRA), en un ecosistema de alta montaña. Luna Azul, 44, 80-93. DOI: 10.17151/luaz.2017.44.6. Recuperado

de http://200.21.104.25/lunazul/index.php/component/content/article?id=222

Esta obra está bajo una Licencia de Creative Commons Reconocimiento CC BY



